\title{
Price-Maker Wind Power Producer Participating in a Joint Day-Ahead and Real-Time Market
}

Delikaraoglou, Stefanos; Papakonstantinou, Athanasios; Ordoudis, Christos; Pinson, Pierre

Published in:

Proceedings of $12<$ sup $>$ th $</$ sup $>$ IEEE International Conference European Energy Market 2015

Link to article, DOI:

10.1109/EEM.2015.7216701

Publication date:

2015

Document Version

Peer reviewed version

Link back to DTU Orbit

Citation (APA):

Delikaraoglou, S., Papakonstantinou, A., Ordoudis, C., \& Pinson, P. (2015). Price-Maker Wind Power Producer Participating in a Joint Day-Ahead and Real-Time Market. In Proceedings of 12 IEEE International Conference European Energy Market 2015 IEEE. https://doi.org/10.1109/EEM.2015.7216701

\section{General rights}

Copyright and moral rights for the publications made accessible in the public portal are retained by the authors and/or other copyright owners and it is a condition of accessing publications that users recognise and abide by the legal requirements associated with these rights.

- Users may download and print one copy of any publication from the public portal for the purpose of private study or research.

- You may not further distribute the material or use it for any profit-making activity or commercial gain

- You may freely distribute the URL identifying the publication in the public portal 


\title{
Price-Maker Wind Power Producer Participating in a Joint Day-Ahead and Real-Time Market
}

\author{
Stefanos Delikaraoglou, Athanasios Papakonstantinou, Christos Ordoudis and Pierre Pinson \\ Dept. of Electrical Engineering \\ Technical University of Denmark \\ Kgs. Lyngby 2800 \\ \{stde, athpapa, chror, ppin\} @ elektro.dtu.dk
}

\begin{abstract}
The large scale integration of stochastic renewable energy introduces significant challenges for power system operators and disputes the efficiency of the current market design. Recent research embeds the uncertain nature of renewable sources by modelling electricity markets as a two-stage stochastic problem, co-optimizing day-ahead and real-time dispatch. In this framework, we introduce a bilevel model to derive the optimal bid of a strategic wind power producer acting as price-maker both in day-ahead and real-time stages. The proposed model is a Mathematical Program with Equilibrium Constraints (MPEC) that is reformulated as a single-level Mixed-Integer Linear Program (MILP), which can be readily solved. Our analysis shows that adopting strategic behaviour may improve producer's expected profit as the share of wind power increases. However, this incentive diminishes in power systems where available flexible capacity is high enough to ensure an efficient market operation.

Index Terms-Electricity markets, mathematical program with equilibrium constraints (MPEC), price-maker, stochastic programming, wind power.
\end{abstract}

\section{NOMENCLATURE}

\section{Sets}

$I \quad$ Set of indices of dispatchable units $i$.

$W \quad$ Set of indices of wind power units $w$

$K \quad$ Set of indices of wind power scenarios $k$.

$J \quad$ Set of indices of bid discretization blocks $j$.

\section{Parameters}

$D \quad$ System demand [MW].

$C_{i} \quad$ Day-ahead offer price of unit $i[\$ / \mathrm{MWh}$.

$C_{i}^{+}, C_{i}^{-} \quad$ Up/down regulation offer price of unit $i$ [\$/MWh].

$C^{\text {sh }} \quad$ Value of involuntarily shed load [\$/MWh].

$P_{i}^{\max }, P_{w}^{\text {cap }} \quad$ Capacity of units $i$ and $w[\mathrm{MW}]$.

$P_{i}^{\min } \quad$ Minimum generation of unit $i$ [MW].

$P_{w k}^{*}$

$R_{i}^{+}, R_{i}^{-}$

Wind power realization in scenario $k$ [MW].

Maximum up/down reserve offered by unit $i$ [MW].

\section{Variables}

$p_{w}^{\text {bid }}$

$p_{i}, p_{w}$

$r_{i k}^{+}, r_{i k}^{-}$

$p_{w k}^{\text {spill }}$

Bidding quantity of unit $w$ [MW].

Day-ahead dispatch of units $i$ and $w$ [MW].

$\mathrm{Up} /$ down regulation by unit $i$ in scenario $k$ [MW].

Wind power spilled by unit $w$ in scenario $k$ [MW].

Load shedding in scenario $k$ [MW]

\section{INTRODUCTION}

The current setup of the European electricity market is based on sequential clearing of two main trading floors. The dayahead (forward) market is cleared 12-36 hours before actual operation based on the expected values of uncertain parameters, e.g., wind power production. To maintain system balance during real-time operation, any deviations from the day-ahead dispatch are compensated by the activation of reserves in the balancing market. This market architecture was considered adequate to cope with the limited and discrete uncertainty arising from load deviations and equipment contingencies, respectively. However, such market designs are challenged as the share of non-dispatchable and partly predictable renewables increases in the power system. To cope effectively with this uncertainty, system operators and wind power producers have to consider novel market designs and trading strategies to mitigate their exposure to increased system costs and financial losses induced by the deployment of expensive reserves.

To this end, we follow recent research focusing on market setups which jointly optimize day-ahead dispatch and realtime reserve deployment in a single settlement energy-only market [1], [2]. Having a probabilistic description of system uncertainties in form of scenarios, the market-clearing model is formulated as a two-stage stochastic programming problem where the first stage represents the day-ahead schedule and the second stage represents the real-time dispatch, i.e., the decisions which can be modified once uncertainty is revealed. As opposed to existing market designs which do not allow for any inter-temporal arbitrage to deal with uncertainty, the stochastic market-clearing can pre-position available generation according to its technical and economic characteristics to secure adequate flexible capacity during real-time operation.

A direct consequence of this advanced market architecture is that support mechanisms (e.g., feed-in tariffs, exemption from imbalance costs) aimed to safeguard wind power producers against the volatility of electricity prices may become obsolete. Such mechanisms are gradually removed as this generation technology reaches grid parity, enforcing wind power producers to trade their production in electricity markets according to the same rules that apply to conventional generators. Hence, a strategic wind power producer may consider minimizing its exposure to the risk associated with forecast uncertainty by accounting for its potential recourse actions. Relevant literature [3], [4] has focused on optimal bidding strategies under the assumption of a price-taker wind power producer. However, the soundness of this argument diminishes in areas where wind power has a dominant position in the market [5], which allows it to act as a price-maker. In that vein, recent works modelled the participation of a price-maker wind power producer either in forward [6] or in real-time [7] markets. 
To our knowledge, this research is the first to study a pricemaker wind power producer's optimal bid in a co-optimized day-ahead and real-time market cleared using stochastic programming. We contribute to the state-of-the-art by considering the impact of a wind power producer's strategic behaviour simultaneously on both market settlements as opposed to previous studies. We develop a methodological framework to derive the optimal bid of a price-maker wind power producer based on a stochastic bilevel model. Applying the proposed method on a case study, we show that as wind power stakes increase, a wind power producer expects to increase its profit by strategic bidding. On the contrary, as the flexible capacity offered by dispatchable generator increases, the wind power producer derives no additional gains through strategic bidding.

The rest of the paper is organized as follows: In Section II we discuss the setup of the problem while in Section III we introduce the mathematical formulation. In Section IV we present and discuss the results of a relevant case study. Finally, Section V concludes the paper.

\section{Wind PoWer Producer AS A PRICE-MAKeR UNDER UNCERTAINTY}

In this section we provide the general framework upon which we build a bilevel model accounting for the Stackelberg relationship between wind power producers (leaders) and market operators (followers). First, we model stochastic production and then proceed to introduce a set of basic assumptions consistent with literature on this topic.

We consider that a wind power producer faces an upper limit $P_{w}^{\text {cap }}$ in its output defined by the technical specifications of the deployed wind turbines $w$ and that the real-time generation is equal to $y P_{w}^{\text {cap }}$, where $y \in[0,1]$ is the realization of the random variable $Y$ which models wind power stochastic output. In consistence with stochastic programming literature, the electricity production from a stochastic producer can be modelled by a finite set $K$ of scenarios sampled from the aforementioned random variable. The predicted wind power realization for scenario $k \in K$ is denoted by $P_{w k}^{*}$.

Now, regarding the set of assumptions, the proposed setup does not consider network constraints. This is consistent with the zonal pricing scheme of the European electricity markets, e.g., NordPool. We only take into account wind power uncertainty during real-time operation. However, note that the model can be extended including network representation and other sources of uncertainty. In the absence of inter-temporal coupling constraints between the trading periods, e.g., ramping limits, we focus on a single time period, while social welfare maximization is equivalent to cost minimization given the assumption of completely inelastic demand.

Finally, we consider that only a single wind power producer may exhibit strategic behaviour, while the rest of market participants are perfectly competitive, i.e, offer their full capacity at marginal cost. This is a relevant assumption in regions where a few stakeholders operate the majority of wind power plants. Nevertheless, it should be noted that the extension to multiple price-maker wind power producers can be formulated as a stochastic Equilibrium Program with Equilibrium Constraints (EPEC) modelling the multiple-leader-follower problem. This topic is left for future research. Moreover, in the absence of fuel costs, the selling offer price of wind power is set equal to zero and thus the only strategic decision parameter of the producer is the overall capacity offered in the dayahead market, while the probabilistic information about its production is centrally obtained. Future work could focus on these forecasts being additional strategic components for wind power producers.

\section{MAThematical Formulation}

In Section III-A we formulate the bilevel optimization problem which determines the wind power producer's optimal offer. In Section III-B the bilevel problem is transformed into a single-level Mathematical Program with Equilibrium Constraints (MPEC), which is then turned into a Mixed-Integer Linear Program (MILP) in Section III-C.

\section{A. Bilevel model}

A strategic producer owing $w$ wind farms derives the optimal bidding quantity employing the following bilevel model:

$$
\begin{aligned}
& \operatorname{Max}_{p_{w}^{\text {bid }}} \lambda^{\text {DA }} \sum_{w \in W} p_{w}+\sum_{k \in K}\left[\lambda_{k}^{\text {B }} \sum_{w \in W}\left(P_{w k}^{*}-p_{w}-p_{w k}^{\text {spill }}\right)\right] \\
& p_{w}^{\text {bid }} \leq P_{w}^{\text {cap }}, \quad \forall w
\end{aligned}
$$

subject to

$$
\begin{aligned}
& p_{w}, p_{w k}^{\text {spill }}, \lambda^{\mathrm{DA}}, \lambda_{k}^{\mathrm{B}} \in \arg \{ \\
& \underset{\Xi}{\operatorname{Min} .} \sum_{i \in I} C_{i} p_{i}+\mathbb{E}_{k}\left[\sum_{i \in I}\left(C_{i}^{+} r_{i k}^{+}-C_{i}^{-} r_{i k}^{-}\right)+C^{\mathrm{sh}} l_{k}^{\mathrm{sh}}\right]
\end{aligned}
$$

subject to

$$
\begin{aligned}
& \sum_{w \in W} p_{w}+\sum_{i \in I} p_{i}=D: \lambda^{\mathrm{DA}} \\
& p_{i} \leq P_{i}^{\mathrm{max}}: \bar{\mu}_{i}^{\mathrm{P}}, \quad \forall i \\
& P_{i}^{\mathrm{min}} \leq p_{i}: \underline{\mu}_{i}^{\mathrm{P}}, \quad \forall i \\
& p_{w} \leq p_{w}^{\mathrm{bid}}: \bar{\rho}_{w}^{\mathrm{P}}, \quad \forall w \\
& 0 \leq p_{w}: \underline{\rho}_{w}^{\mathrm{P}}, \quad \forall w \\
& \sum_{i \in I}\left(r_{i k}^{+}-r_{i k}^{-}\right)+l_{k}^{\mathrm{sh}} \\
& +\sum_{w \in W}\left(P_{w k}^{*}-p_{w}-p_{w k}^{\mathrm{spill}}\right)=0: \lambda_{k}^{\mathrm{B}}, \quad \forall k \\
& p_{i}+r_{i k}^{+} \leq P_{i}^{\mathrm{max}}: \bar{\mu}_{i k}^{\mathrm{PR}}, \quad \forall i, \forall k \\
& p_{i}-r_{i k}^{-} \geq P_{i}^{\min }: \underline{\mu}_{i k}^{\mathrm{PR}}, \quad \forall i, \forall k \\
& r_{i k}^{+} \leq R_{i}^{+}: \bar{\mu}_{i k}^{\mathrm{R}}, \quad \forall i, \forall k \\
& r_{i k}^{-} \leq R_{i}^{-}: \bar{\mu}_{i k}^{\mathrm{R}}, \quad \forall i, \forall k \\
& r_{i k}^{+}, r_{i k}^{-} \geq 0:\left(\underline{\mu}_{i k}^{\mathrm{R}}, \underline{\mu}_{i k}^{\mathrm{R}}\right), \quad \forall i, \forall k \\
& l_{k}^{\mathrm{sh}} \leq D: \bar{\mu}_{k}^{\mathrm{sh}}, \quad \forall k \\
& p_{w k}^{\text {spill }} \leq P_{w k}^{*}: \bar{\mu}_{w k}^{\mathrm{spill}}, \quad \forall w, \forall k \\
& \left.0 \leq p_{w k}^{\text {spill }}, l_{k}^{\mathrm{sh}}:\left(\underline{\mu}_{w k}^{\mathrm{spill}}, \quad \underline{\mu}_{k}^{\mathrm{sh}}\right), \quad \forall w, \forall k\right\}
\end{aligned}
$$


where $\mathbb{E}_{k}[\cdot]$ is the expectation operator, defined as the sum over a finite set of scenarios $K$ weighted by the probability of occurrence $\pi_{k}$. The dual variable of each constraint is indicated after a colon. The set of optimization variables of the lower level problem is defined as $\Xi=$ $\left\{p_{w}, p_{i}, r_{i k}^{+}, r_{i k}^{-}, l_{k}^{\text {sh }}, p_{w k}^{\text {spill }}, \lambda^{\mathrm{DA}}, \bar{\mu}_{i}^{\mathrm{P}}, \underline{\mu}_{i}^{\mathrm{P}}, \bar{\rho}_{w}^{\mathrm{P}}, \underline{\rho}_{w}^{\mathrm{P}}, \lambda_{k}^{\mathrm{B}}, \bar{\mu}_{i k}^{\mathrm{PR}}, \underline{\mu}_{i k}^{\mathrm{PR}}\right.$, $\left.\bar{\mu}_{i k}^{\mathrm{R}^{+}}, \mu_{i k}^{\mathrm{R}^{+}}, \bar{\mu}_{i k}^{\mathrm{R}^{-}}, \underline{\mu}_{i k}^{\mathrm{R}^{-}}, \bar{\mu}_{k}^{\mathrm{sh}}, \underline{\mu}_{k}^{\mathrm{sh}}, \bar{\mu}_{w k}^{\text {spill }}, \underline{\mu}_{w k}^{\mathrm{spill}}, \forall i, w, k\right\}$.

The objective function (1a) of the upper level problem maximizes the expected profit of the wind power producer and consists of two terms:

1) The term $\lambda^{\mathrm{DA}} p_{w}$ represents the revenue of each wind farm $w$ in the day-ahead market, calculated as the product of the day-ahead market price $\lambda^{\mathrm{DA}}$ times the scheduled wind power $p_{w}$.

2) The term $\lambda_{k}^{\mathrm{B}}\left(P_{w k}^{*}-p_{w}-p_{w k}^{\text {spill }}\right)$ represents the cost/profit in scenario $k$ for wind farm $w$ participating in the balancing market to settle its deviation from the dayahead schedule. Positive deviations (excess generation) are paid at a price $\lambda_{k}^{\mathrm{B}}$, while negative deviations (generation shortage) are charged at a price $\lambda_{k}^{\mathrm{B}}$.

Note that $\lambda_{k}^{\mathrm{B}}$ are probability weighted balancing market prices, i.e., if a specific scenario $k$ realizes the corresponding real-time price will be equal to $\lambda_{k}^{\mathrm{B}} / \pi_{k}$. Constraint (1b) indicates that the wind power production offered in the day-ahead market is limited by the installed capacity $P_{w}^{\text {cap }}$.

The lower level problem represents the joint clearing of day-ahead and real-time markets, where the objective function (2a) minimizes the expected system cost. Equality constraints (2b) and (2g) guarantee the power balance between production and consumption in day-ahead and real-time operation, respectively. Constraints (2c)-(2f) impose the capacity limits of conventional and wind power units in the forward marketclearing. Moreover, the set of constraints (2h)-(2l) model the power and reserve capacity limits during real-time operation. Finally, the upper and lower bounds of load shedding and wind spillage are enforced by constraints $(2 \mathrm{~m})-(2 \mathrm{o})$.

\section{B. $M P E C$}

Considering that the hierarchical relationship between the two optimization problems cannot be handled directly by available optimization solvers, the bilevel problem is transformed into a single-level problem. Taking into account that the lower level problem (2a)-(2o) is linear, it can be replaced by its KKT conditions [8] as follows

$$
\underset{\Xi^{\dagger}}{\operatorname{Max}} \lambda^{\mathrm{DA}} \sum_{w \in W} p_{w}+\sum_{k \in K}\left[\lambda_{k}^{\mathrm{B}} \sum_{w \in W}\left(P_{w k}^{*}-p_{w}-p_{w k}^{\text {spill }}\right)\right]
$$

subject to

$$
\text { constraints (2b)-(2o) }
$$

$$
\begin{aligned}
& C_{i}-\lambda^{D A}+\bar{\mu}_{i}^{\mathrm{P}}-\underline{\mu}_{i}^{\mathrm{P}}+\sum_{k \in K}\left(\bar{\mu}_{i k}^{\mathrm{PR}}-\underline{\mu}_{i k}^{\mathrm{PR}}\right)=0, \quad \forall i \\
& -\lambda^{\mathrm{DA}}+\bar{\rho}_{w}^{\mathrm{P}}-\underline{\rho}_{w}^{\mathrm{P}}+\sum_{k \in K} \lambda_{k}^{\mathrm{B}}=0, \quad \forall w \\
& \pi_{k} C_{i}^{+}-\lambda_{k}^{\mathrm{B}}+\bar{\mu}_{i k}^{\mathrm{PR}}+\bar{\mu}_{i k}^{\mathrm{R}^{+}}-\underline{\mu}_{i k}^{\mathrm{R}^{+}}=0, \quad \forall i, \forall k
\end{aligned}
$$

$$
\begin{aligned}
& -\pi_{k} C_{i}^{-}+\lambda_{k}^{\mathrm{B}}+\underline{\mu}_{i k}^{\mathrm{PR}}+\bar{\mu}_{i k}^{\mathrm{R}^{-}}-\underline{\mu}_{i k}^{\mathrm{R}^{-}}=0, \quad \forall i, \forall k \\
& \pi_{k} C^{\mathrm{sh}}-\lambda_{k}^{\mathrm{B}}+\bar{\mu}_{k}^{\mathrm{sh}}-\underline{\mu}_{k}^{\mathrm{sh}}=0, \quad \forall k \\
& \lambda_{k}^{B}+\bar{\mu}_{w k}^{\mathrm{sill}}-\underline{\mu}_{w k}^{\mathrm{spill}}=0, \quad \forall w, \forall k \\
& 0 \leq \bar{\mu}_{i}^{\mathrm{P}} \perp P_{i}^{\mathrm{max}}-p_{i} \geq 0, \quad \forall i \\
& 0 \leq \underline{\mu}_{i}^{\mathrm{P}} \perp p_{i}-P_{i}^{\mathrm{min}} \geq 0, \quad \forall i \\
& 0 \leq \bar{\rho}_{w}^{\mathrm{P}} \perp p_{w}^{\text {bid }}-p_{w} \geq 0, \quad \forall w \\
& 0 \leq \underline{\rho}_{w}^{\mathrm{P}} \perp p_{w} \geq 0, \quad \forall w \\
& 0 \leq \bar{\mu}_{i k}^{\mathrm{PR}} \perp P_{i}^{\mathrm{max}}-p_{i}-r_{i k}^{+} \geq 0, \quad \forall i, \forall k \\
& 0 \leq \underline{\mu}_{i k}^{\mathrm{PR}} \perp p_{i}-r_{i k}^{-}-P_{i}^{\text {min }} \geq 0, \quad \forall i, \forall k \\
& 0 \leq \bar{\mu}_{i k}^{\mathrm{R}^{+}} \perp R_{i}^{+}-r_{i k}^{+} \geq 0, \quad \forall i, \forall k \\
& 0 \leq \underline{\mu}_{i k}^{\mathrm{R}^{+}} \perp r_{i k}^{+} \geq 0, \quad \forall i, \forall k \\
& 0 \leq \bar{\mu}_{i k}^{\mathrm{R}^{-}} \perp R_{i}^{-}-r_{i k}^{-} \geq 0, \quad \forall i, \forall k \\
& 0 \leq \underline{\mu}_{i k}^{\mathrm{R}^{-}} \perp r_{i k}^{-} \geq 0, \quad \forall i, \forall k \\
& 0 \leq \bar{\mu}_{k}^{\text {sh }} \perp D-l_{k}^{\text {sh }} \geq 0, \quad \forall k \\
& 0 \leq \underline{\mu}_{k}^{\text {sh }} \perp l_{k}^{\text {sh }} \geq 0, \quad \forall k \\
& 0 \leq \bar{\mu}_{w k}^{\text {spill }} \perp P_{w k}^{*}-p_{w k}^{\text {spill }} \geq 0, \quad \forall w, \forall k \\
& 0 \leq \underline{\mu}_{w k}^{\text {spill }} \perp p_{w k}^{\text {spill }} \geq 0, \quad \forall w, \forall k
\end{aligned}
$$

where $p_{w}^{\text {bid }} \cup \Xi=\Xi^{\dagger}$ and the $\perp$ operator indicates that the product of the expressions on its right and left side is equal to zero. Equations (3c)-(3h) are stationarity conditions while (3i)-(3v) are complementarity slackness conditions.

\section{MILP}

The MPEC problem (3) described in the previous section cannot be readily solved due to the bilinear terms $\lambda^{\mathrm{DA}} p_{w}$ and $\lambda_{k}^{\mathrm{B}} p_{w}$ that appear in the objective function, and the nonlinear complementarity constraints (3i)-(3v). These nonlinearities are raised by the following set of transformations.

The complementarity conditions (3k), (3l) and (3u), (3v) write as

$$
\begin{aligned}
& \bar{\rho}_{w}^{\mathrm{P}}\left(p_{w}^{\text {bid }}-p_{w}\right)=0 \Leftrightarrow \bar{\rho}_{w}^{\mathrm{P}} p_{w}^{\text {bid }}=\bar{\rho}_{w}^{\mathrm{P}} p_{w}, \quad \forall w \\
& \underline{\rho}_{w}^{\mathrm{P}} p_{w}=0, \quad \forall w \\
& \bar{\mu}_{w k}^{\text {sill }}\left(P_{w k}^{*}-p_{w k}^{\text {spill }}\right) \Leftrightarrow \bar{\mu}_{w k}^{\text {spill }} P_{w k}^{*}=\bar{\mu}_{w k}^{\text {spill }} p_{w k}^{\text {spill }}, \quad \forall w, \forall k \\
& \underline{\mu}_{w k}^{\text {spill }} p_{w k}^{\text {spill }}=0, \quad \forall w, \forall k
\end{aligned}
$$

Constraint (3d) can be reformulated as

$$
\lambda^{\mathrm{DA}}-\sum_{k \in K} \lambda_{k}^{\mathrm{B}}=\bar{\rho}_{w}^{\mathrm{P}}-\underline{\rho}_{w}^{\mathrm{P}}, \quad \forall w
$$

while constraint (3h) can be written as

$$
\bar{\mu}_{w k}^{\text {spill }}-\underline{\mu}_{w k}^{\text {spill }}=-\lambda_{k}^{\mathrm{B}}, \quad \forall w, k
$$

Multiplying equation (4f) by $p_{w k}^{\text {spill }}$, we obtain

$$
\bar{\mu}_{w k}^{\text {spill }} p_{w k}^{\text {spill }}-\underline{\mu}_{w k}^{\text {spill }} p_{w k}^{\text {spill }}=-\lambda_{k}^{\mathrm{B}} p_{w k}^{\text {spill }}, \quad \forall w, k
$$

Then, the objective function (3a) can be reformulated as

$$
\lambda^{\mathrm{DA}} \sum_{w \in W} p_{w}+\sum_{k \in K}\left[\lambda_{k}^{\mathrm{B}} \sum_{w \in W}\left(P_{w k}^{*}-p_{w}-p_{w k}^{\text {spill }}\right)\right]=
$$




$$
\begin{aligned}
& \sum_{w \in W} p_{w}\left[\lambda^{\mathrm{DA}}-\sum_{k \in K} \lambda_{k}^{\mathrm{B}}\right]+\sum_{k \in K} \lambda_{k}^{\mathrm{B}} \sum_{w \in W}\left(P_{w k}^{*}-p_{w k}^{\mathrm{spill}}\right) \underset{(4 \mathrm{~g})}{\left(\frac{4 \mathrm{e}}{(4)}\right.} \\
& \sum_{w \in W} p_{w}\left(\bar{\rho}_{w}^{\mathrm{P}}-\underline{\rho}_{w}^{\mathrm{P}}\right)+\sum_{k \in K} \lambda_{k}^{\mathrm{B}} \sum_{w \in W} P_{w k}^{*} \\
& +\sum_{k \in K} \sum_{w \in W}\left(\bar{\mu}_{w k}^{\text {spill }} p_{w k}^{\text {spill }}-\underline{\mu}_{w k}^{\text {spill }} p_{w k}^{\text {spill }}\right) \underset{(4 \mathrm{~d})}{(4 \mathrm{~b})} \\
& \sum_{w \in W} p_{w} \bar{\rho}_{w}^{\mathrm{P}}+\sum_{k \in K} \lambda_{k}^{\mathrm{B}} \sum_{w \in W} P_{w k}^{*}+\sum_{k \in K} \sum_{w \in W} \bar{\mu}_{w k}^{\text {spill }} p_{w k}^{\text {spill }} \underset{(4 \mathrm{c})}{\overline{(4 \mathrm{a})}} \\
& \sum_{w \in W} p_{w}^{\mathrm{bid}} \bar{\rho}_{w}^{\mathrm{P}}+\sum_{k \in K} \lambda_{k}^{\mathrm{B}} \sum_{w \in W} P_{w k}^{*}+\sum_{k \in K} \sum_{w \in W} \bar{\mu}_{w k}^{\text {spill }} P_{w k}^{*}
\end{aligned}
$$

The final expression of (4h) still involves the nonlinear term $p_{w}^{\mathrm{bid}} \bar{\rho}_{w}^{\mathrm{P}}$. However, assuming that the bidding quantity $p_{w}^{\mathrm{bid}}$ can be discretized in $N^{J}$ equal blocks as

$$
\begin{aligned}
& p_{w}^{\text {bid }}=\sum_{j \in J} v_{w j} \hat{p}_{w} \\
& P_{w}^{\text {cap }}=N^{J} \hat{p}_{w}
\end{aligned}
$$

where $v_{w j}$ is a binary variable and $\hat{p}_{w}$ is the block size, e.g., $1 \mathrm{MW}$, the term $p_{w}^{\mathrm{bid}} \bar{\rho}_{w}^{\mathrm{P}}$ can be written as

$$
p_{w}^{\mathrm{bid}} \bar{\rho}_{w}^{\mathrm{P}}=\bar{\rho}_{w}^{\mathrm{P}} \sum_{j \in J} v_{w j} \hat{p}_{w}=\hat{p}_{w} \sum_{j \in J} v_{w j} \bar{\rho}_{w}^{\mathrm{P}}
$$

The term $z_{w j}=v_{w j} \bar{\rho}_{w}^{\mathrm{P}}$ is a product of binary $v_{w j}$ and continuous variable $\bar{\rho}_{w}^{\mathrm{P}} \in\left[\bar{\rho}_{w, \text { min }}^{\mathrm{P}}, \bar{\rho}_{w, \text { max }}^{\mathrm{P}}\right]$, where $\bar{\rho}_{w, \text { min }}^{\mathrm{P}}, \bar{\rho}_{w, \text { max }}^{\mathrm{P}}$ are constants. This product can be linearised [9] as

$$
\begin{aligned}
& \bar{\rho}_{w, \min }^{\mathrm{P}} v_{w j} \leq z_{w j} \leq \bar{\rho}_{w, \text { max }}^{\mathrm{P}} v_{w j} \\
& \bar{\rho}_{w}^{\mathrm{P}}-\bar{\rho}_{w, \max }^{\mathrm{P}}\left(1-v_{w j}\right) \leq z_{w j} \leq \bar{\rho}_{w}^{\mathrm{P}}-\bar{\rho}_{w, \min }^{\mathrm{P}}\left(1-v_{w j}\right)
\end{aligned}
$$

Hence, the objective function (4h) is now written as

$$
\operatorname{Max}_{\Xi^{\dagger} \cup \Xi^{\ddagger}} \sum_{w \in W} \hat{p}_{w} \sum_{j \in J} z_{w j}+\sum_{k \in K} \sum_{w \in W}\left(\lambda_{k}^{\mathrm{B}}+\bar{\mu}_{w k}^{\mathrm{spill}}\right) P_{w k}^{*}
$$

where $\Xi^{\ddagger}$ contains the auxiliary variables needed to apply transformations (4la)-(4lb) and to reformulate complementarity relationships (3i)-(3v) via disjunctive constraints [10].

\section{Numerical Results ANd Discussion}

In this section we evaluate the proposed model using a power system based on a modified version of the IEEE Reliability Test System presented in [11]. We adapted the power system to fit our specific assumptions, most notably the lack of network structure and the existence of a single wind power producer. The conventional generators with the same power output capacity and offer costs were merged and the total capacity of the system was scaled down by a factor of five. Table I presents the data for the conventional units. Up and down regulating costs set equal to 1.1 and 0.91 of the energy offer price, respectively. The total system demand is equal to $450 \mathrm{MW}$. Wind power production uncertainty is modelled using a Beta distribution $B(\alpha, \beta)$ in consistence with relevant literature [12]. Wind power uncertainty is described using a set of 50 equiprobable scenarios sampled from a Beta distribution with parameters $\alpha=2.1725$ and $\beta=1.7775$. The
TABLE I

CONVENTIONAL Unit DATA

\begin{tabular}{ccccccccc}
\hline Unit & G1 & G2 & G3 & G4 & G5 & G6 & G7 & G8 \\
\hline$C_{i}$ & 15.97 & 22.72 & 22.13 & 30.4 & 5.66 & 0 & 11.26 & 11.72 \\
$P_{i}^{\max }$ & 60.8 & 60 & 118.2 & 12 & 160 & 60 & 124 & 70 \\
$C_{i}^{+}$ & 0 & 0 & 24.34 & 33.44 & 0 & 0 & 0 & 0 \\
$C_{i}^{-}$ & 0 & 0 & 20.13 & 27.66 & 0 & 0 & 0 & 0 \\
$R_{i}^{+}$ & 0 & 0 & 71.49 & 7.26 & 0 & 0 & 0 & 0 \\
$R_{i}^{-}$ & 0 & 0 & 71.49 & 7.26 & 0 & 0 & 0 & 0 \\
\hline
\end{tabular}

spilling action is considered cost free, whereas $C^{\text {sh }}$ is equal to $200 \$ / \mathrm{MWh}$ and $P_{i}^{\min }$ is set to zero for all units.

Fig. 1 compares the evolution of the expected profit as a function of wind power penetration for a strategic versus a non-strategic wind power producer. In the current context, a non-strategic producer offers all its capacity $P_{w}^{\text {cap }}$ in the dayahead market. The general trend of these curves indicates that larger volumes of wind power generation lead to higher expected profits. However, moving from $10 \%$ to $15 \%$ wind penetration results into a moderate decrease of the expected profits due to the market dynamics. In that case, the higher wind power in-feed changes the marginal unit of the system and consequently entails a day-ahead price reduction which cannot be compensated by the larger traded quantity. For penetration levels up to $25 \%$, both trading strategies achieve identical profits suggesting that the wind power producer is not able to improve its position by hedging the forecast uncertainty between the day-ahead and real-time markets. On the contrary, for shares greater than $30 \%$, wind power becomes a pricemaker and thus its bidding strategy can influence the marketclearing and enhance its economic performance.

In the current case study where any real-time imbalances depend solely on the deviations of the single producer from the day-ahead schedule, the optimal bid is driven by the cost of re-dispatching actions. Considering that all wind power deviations, irrespective of sign, are penalized in real-time market, i.e., production surplus (deficit) is sold (charged) in lower (higher) price than the forward price, the strategic producer aims to protect himself against extreme imbalance costs. Hence, it is relevant to take into account the nonsymmetric regulation unit costs of this market setup, i.e., the positive/negative price premium from the day-ahead price for up/down regulation. To better understand this concept, one may consider the difference in cost between the most expensive up and down re-dispatching actions; namely load shedding and wind spillage, respectively. Hence, the strategic producer will aim primarily to reduce its exposure to extreme up-regulation costs by taking a long position $\left(p_{w}^{\text {bid }}<P_{w k}^{*}\right)$ as wind penetration increases. This justifies the fact that the strategic offering achieves higher profitability for larger shares of wind, where the probability of the system resorting to expensive re-dispatching actions is higher. Nonetheless, improving the expected profit of a strategic producer does not translate to a proportional system cost increase. For example, at penetration levels ranging from $40 \%$ to $50 \%$, the strategic producer achieves more than $7 \%$ higher expected profit, while the corresponding system cost raises less than $1 \%$. 


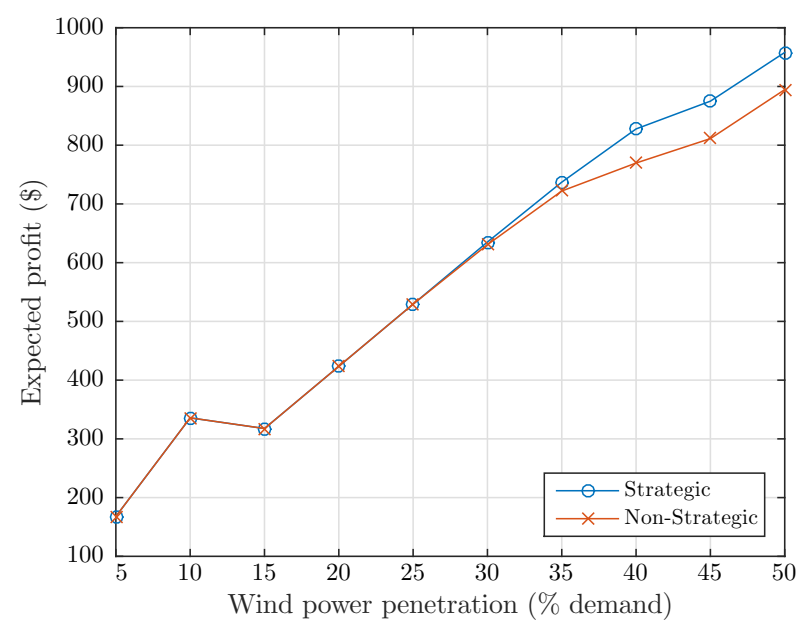

Fig. 1. Expected profit of wind power producer as a function of wind power penetration.

Following the preceding analysis, we study the effect of available flexible capacity, i.e., generation providing balancing services, to the profit of the wind power producer for a penetration level of 35\%. Fig. 2 demonstrates the positive impact of system flexibility on the profitability of wind. This can be explained considering that higher flexibility translates into a less penalizing balancing market in a sense that it reduces the need for very expensive re-dispatching actions in extreme wind scenarios. Nevertheless, for flexible capacity greater than $22.5 \%$ we notice a saturation of the expected profit, while the corresponding curves for both offering strategies coincide. This is an indication of an efficient market managing to align the interests of the system operator and the strategic producer, since the latter has no incentive to deviate from the day-ahead dispatch that minimizes system cost.

The optimal offering problem has been solved using CPLEX 12.6 under GAMS. The average computation time of each problem was 20 seconds on a stationary computer with Intel i7 4-core processor clocking at $3.4 \mathrm{GHz}$ and $8 \mathrm{~GB}$ of RAM.

\section{CONCLusions And Future Work}

This paper considers the trading strategy of a wind power producer participating in a co-optimized day-ahead and realtime market-clearing. The hierarchical relationship between the decisions of a price-maker producer and the market outcome is modelled as an MPEC and reformulated as an MILP. Our case study shows that in view of its uncertainty risk and the potential recourse actions, a strategic producer with dominant market position may improve its expected profits by adjusting its day-ahead quantity offer. However, this behaviour can be offset by increasing the flexible capacity of the system.

For future research we intend to include network structure and gradually relax the assumptions related to the number of wind power producers in the system. This will allow to access the market value and the strategic positioning of a pricemaker producer not only with respect to its size but also to its correlation with the aggregate wind power production in

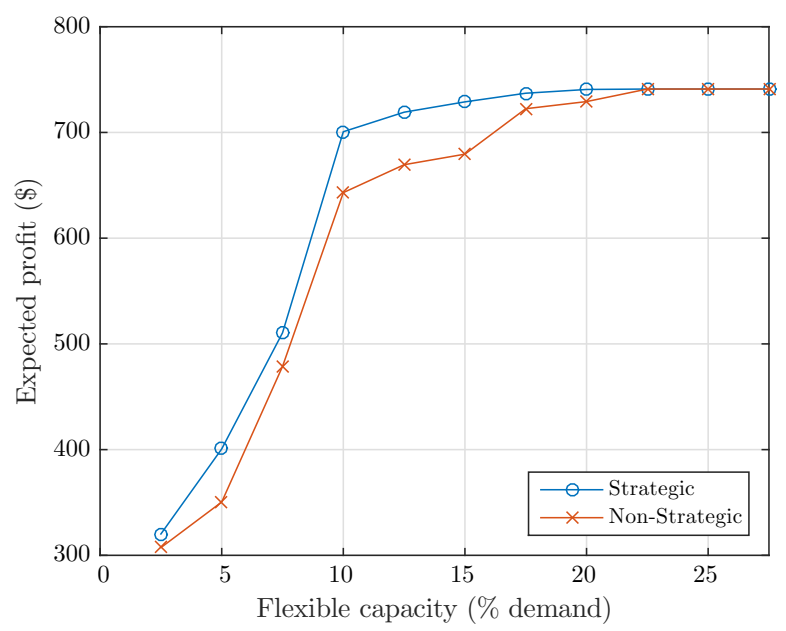

Fig. 2. Expected profit of wind power producer as a function of flexible capacity ranging from $2.5 \%$ to $27.5 \%$ for $35 \%$ wind power penetration.

the system. This setup can be further extended by introducing competition among multiple strategic producers following an EPEC approach to determine the market equilibrium.

\section{ACKNOWLEDGEMENT}

Stefanos Delikaraoglou is funded by Energinet.dk in support of ERA-Net SmartGrids through project "BPES-Balancing Power in the European System", No. 2010-1-10816. The other authors are supported by the Danish Strategic Research Council (DSF) through projects "5s-Future Electricity Markets", No. 12-132636/DSF and "CITIES", No. 1305-00027B/DSF.

\section{REFERENCES}

[1] G. Pritchard, G. Zakeri, and A. Philpott, "A single-settlement, energyonly electric power market for unpredictable and intermittent participants," Oper. Res., vol. 58, no. 4, pp. 1210-1219, 2010.

[2] J. M. Morales, A. J. Conejo, K. Liu, and J. Zhong, "Pricing electricity in pools with wind producers," IEEE Trans. Power Syst., vol. 27, no. 3, pp. 1366-1376, 2012

[3] P. Pinson, C. Chevallier, and G. N. Kariniotakis, "Trading wind generation from short-term probabilistic forecasts of wind power," IEEE Trans. Power Syst., vol. 22, no. 3, pp. 1148-1156, 2007.

[4] J. M. Morales, A. J. Conejo, and J. Pérez-Ruiz, "Short-term trading for a wind power producer," IEEE Trans. Power Syst., vol. 25, no. 1, pp. 554-564, 2010.

[5] T. Jónsson, P. Pinson, and H. Madsen, "On the market impact of wind energy forecasts," Energy Econ., vol. 32, no. 2, pp. 313 - 320, 2010.

[6] L. Baringo and A. J. Conejo, "Strategic offering for a wind power producer," IEEE Trans. Power Syst., vol. 28, no. 4, pp. 4645-4654, 2013.

[7] M. Zugno, J. M. Morales, P. Pinson, and H. Madsen, "Pool strategy of a price-maker wind power producer," IEEE Trans. Power Syst., vol. 28, no. 3, pp. 3440-3450, 2013.

[8] Z.-Q. Luo, J.-S. Pang, and D. Ralph, Mathematical Programs with Equilibrium Constraints. New York: Cambridge University Press, 1996.

[9] C. A. Floudas, Nonlinear and Mixed-Integer Optimization: Fundamentals and Applications. New York: Oxford University Press, 1995.

[10] J. Fortuny-Amat and B. McCarl, "A representation and economic interpretation of a two-level programming problem," J. Oper. Res. Soc., vol. 32, no. 9, pp. 783-792, 1981.

[11] A. J. Conejo, M. Carrion, and J. M. Morales, Decision Making under Under Uncertainty in Electricity Markets. New York: Springer, 2010.

[12] A. Fabbri, T. Roman, J. Abbad, and V. Quezada, "Assessment of the cost associated with wind generation prediction errors in a liberalized electricity market," IEEE Trans. Power Syst., vol. 20, no. 3, pp. 14401446, 2005. 